\title{
The TRAF3-binding site of human molluscipox virus FLIP molecule MC159 is critical for its capacity to inhibit Fas-induced apoptosis
}

\author{
M Thurau ${ }^{1,2,3}$, H Everett ${ }^{1,2}$, M Tapernoux $^{1}, \mathrm{~J} \mathrm{Tschopp}^{1}$ and \\ M Thome ${ }^{\star, 1}$ \\ 1 Department of Biochemistry, University of Lausanne, BIL Biomedical Research \\ Center, Chemin des Boveresses 155, Epalinges, Switzerland \\ 2 These two authors contributed equally to this work. \\ ${ }^{3}$ Current address: Department for Molecular and Experimental Surgery, Clinical \\ Center, University of Erlangen-Nuremberg, Schwabachanlage 10, D-91054 \\ Erlangen, Germany. \\ * Corresponding author: M Thome, Department of Biochemistry, University of \\ Lausanne, Chemin des Boveresses 155, Epalinges VD CH-1066, Switzerland. \\ Tel: + 41216925737 Fax: + 41216925705 \\ E-mail: Margot.ThomeMiazza@unil.ch
}

Received 22.7.05; revised 15.11.05; accepted 22.11.05; published online 20.1.06 Edited by D Vaux

\begin{abstract}
Members of the viral Flice/caspase-8 inhibitory protein (v-FLIP) family prevent induction of apoptosis by death receptors through inhibition of the processing and activation of procaspase-8 and -10 at the level of the receptor-associated death-inducing signaling complex (DISC). Here, we have addressed the molecular function of the v-FLIP member MC159 of the human molluscum contagiosum virus. MC159 FLIP powerfully inhibited both caspase-dependent and caspase-independent cell death induced by Fas. The Cterminal region of $\mathrm{MC} 159$ bound TNF receptor-associated factor (TRAF)3, was necessary for optimal TRAF2 binding, and mediated the recruitment of both TRAFs into the Fas DISC. TRAF-binding-deficient mutants of MC159 showed impaired inhibition of FasL-induced caspase-8 processing and Fas internalization, and had reduced antiapoptotic activity. Our findings provide evidence that a MC159/TRAF2/ TRAF3 complex regulates a new aspect of Fas signaling, and identify MC159 FLIP as a molecule that targets multiple features of Fas-induced cell death.

Cell Death and Differentiation (2006) 13, 1577-1585.

doi:10.1038/sj.cdd.4401847; published online 20 January 2006
\end{abstract}

Keywords: poxvirus; MCV; FLIP; TRAF; caspase-8; cell death

Abbreviations: CTL, cytotoxic T lymphocyte; DD, death domain; DED, death effector domain; DISC, death-inducing signaling complex; FLIP, Flice/caspase-8 inhibitory protein; MCV, molluscum contagiosum virus; TNF, tumor necrosis factor; TRAF, TNF receptor-associated factor

\section{Introduction}

Fas (CD95) is a member of the tumor necrosis factor (TNF) receptor family that can induce cell death by both caspase- dependent (apoptotic) and caspase-independent mechanisms. ${ }^{1-3}$ The molecular mechanism of apoptotic Fas signaling has been intensively studied. Fas engagement induces recruitment, via the adaptor protein FADD, of procaspase-8 and -10 to form a receptor-bound death-inducing signaling complex (DISC). ${ }^{4}$ This association probably brings the recruited procaspase-8 (or -10) molecules into close proximity at the receptor level, and thereby allows them to become activated by dimerization. ${ }^{5,6}$ Following dimerization to the catalytically active form, the $\mathrm{N}$-terminal death effector domains (DEDs) are removed by intermolecular processing, ${ }^{7}$ leading to the release of active caspase-8 (or -10) into the cytoplasm and subsequently to the direct cleavage and activation of the effector caspase- $3 .^{8}$ An additional Fas signaling pathway has been described, which appears to be predominant in so-called type II cells, that show reduced or delayed DISC formation. ${ }^{9}$ In these cells, only small amounts of caspase- 8 are processed, which may be insufficient to directly activate caspase- 3 , but sufficient to activate the proapoptotic Bcl-2 family member, Bid. ${ }^{4,10,11}$ The truncated Bid cleavage product, tBid, may act to aggregate the proapoptotic Bcl-2 family members Bak or Bax, thereby leading to mitochondrial changes including the loss of inner membrane potential $\left(\Delta \Psi_{\mathrm{m}}\right)$ and cytochrome $c$ release into the cytoplasm. ${ }^{12}$ Cytoplasmic cytochrome $c$ promotes the activation of caspase-9 via the assembly of a cytochrome c/Apaf-1/ caspase-9 complex called the apoptosome. ${ }^{13-15}$ Activated caspase- 9 in turn cleaves and activates caspase-3.

Fas also induces a caspase-independent mechanism of cell death that has morphological features of necrosis. ${ }^{2,16}$ Fasinduced necrosis is insensitive to the pan-caspase inhibitor z-VAD and depends on the kinase activity of RIP $1,{ }^{16}$ a Ser/ Thr kinase originally identified in a two-hybrid screen as a death domain (DD)-containing protein that binds to the Fas DD. ${ }^{17}$ In addition to RIP1, the adaptor protein FADD also plays an essential role in Fas-induced necrosis, since T-cell lines deficient in FADD or expressing a dominant negative form of FADD, containing only the DD, show impaired necrosis. ${ }^{16}$

Fas-mediated cell death plays a critical role in the elimination of activated lymphocytes and also participates in the removal of tumor cells or virally infected cells by FasLexpressing cytotoxic T lymphocytes (CTLs). ${ }^{3,18,19}$ Viruses have evolved a number of molecular strategies designed to inhibit host defenses that are based on promoting the death of virally infected cells. ${ }^{18,20,21}$ Viral homologues of the Flice/ caspase-8-inhibitory proteins (FLIPs) interfere with the recruitment, processing and release of caspase- 8 and -10 induced by Fas and other death receptors, and thereby inhibit one of the earliest steps of death receptor signaling. ${ }^{22}$

Molluscum contagiosum virus (MCV) is a human poxvirus that replicates in keratinocytes in the skin, where it causes small, benign lesions that generally lack signs of inflammation. In immunosuppressed patients, the MCV lesions are often 
verrucous and bigger in size. ${ }^{18,23} \mathrm{MCV}$ encodes two FLIP homologues, called MC159 and MC160, which differ from herpesviral FLIP proteins by their unique C-terminal extensions of 66 and 202 amino acids, respectively. ${ }^{24-26}$ The molecular functions of the two MCV FLIPs, and especially of their unusually long C-terminal extensions, are only partly understood.

Both proteins bind to FADD and caspase-8 via their $\mathrm{N}$-terminal DEDs, but only MC159 efficiently inhibits death receptor-induced apoptosis. ${ }^{24-27}$ Interestingly, the antiapoptotic function of MC159 FLIP appears to be partially independent of its intact DEDs and thus of its capacity to bind FADD and caspase- $8 .{ }^{28}$ Since MCV cannot be grown in culture, it is difficult to study the properties of individual viral proteins in their natural context. ${ }^{29} \mathrm{We}$ therefore decided to use Jurkat cells as a model system to investigate the molecular function of MC159 in the Fas pathway. These cells respond very efficiently to FasL and were used by others to study the antiapoptotic function of MC159. ${ }^{28,30}$ Using this model system, we find that MC159 FLIP inhibits both caspasedependent and caspase-independent cell death induced by Fas. Moreover, we show that MC159 FLIP inhibits Fasinduced apoptosis by blocking both caspase-8 processing and Fas endocytosis, and that an intact TNF receptorassociated factor (TRAF)3-binding site in the unique C-terminus of MC159 FLIP is required for the full antiapoptotic capacity of MC159 FLIP.

\section{Results}

\section{MC159 FLIP fully protects Jurkat cells from apoptotic and necrotic cell death}

To analyze the molecular function of the MC159 FLIP, we initially generated stable MC159-expressing Jurkat clones and tested these for their sensitivity to Fas-induced cell death, which has been previously shown to depend on both caspasedependent (apoptotic) and caspase-independent, RIP1-dependent (necrotic) mechanisms. ${ }^{16,31}$ Fas-mediated cell death was induced using a cytotoxic recombinant FasL construct containing two trimerized FasL molecules, Fc-FasL, which is competent to induce the formation of a DISC. ${ }^{32}$ In four independent clones analyzed, MC159 FLIP provided full protection against FasL-induced cell death (Figure 1), while pretreatment of mock-transfected or parental cells with saturating concentrations of the pan-caspase inhibitor z-VAD provided only partial protection (Figure 1 and data not shown), as previously reported. ${ }^{16}$ Therefore, MC159 FLIP fully protects against both caspase-dependent and caspaseindependent cell death induced by Fas.

\section{The C-terminus of MC159 FLIP binds TRAF3 via two PVQES TRAF-binding motifs}

Since MC159 FLIP differs from the cellular FLIPs and from herpesviral FLIP family members by its unique C-terminal extension, we reasoned that this part of the molecule could have interesting features relevant to the powerful deathprotective properties of MC159 FLIP. Sequence analysis of the C-terminal region of MC159 FLIP revealed the presence of

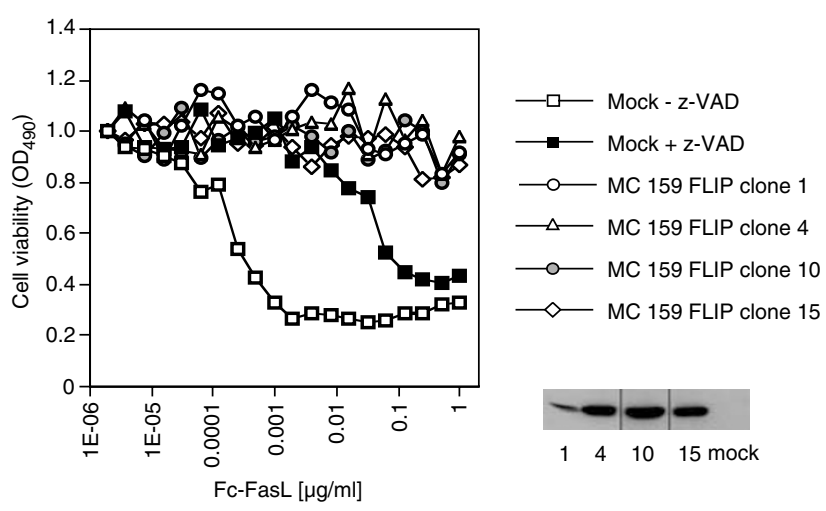

Figure 1 MC159 FLIP completely prevents Fas-induced apoptosis and necrosis. Jurkat clones stably transfected with the indicated FLIP constructs were treated overnight with Fc-FasL and cell viability was assessed using the PMS/ MTS assay. The Western blot shows the expression levels of VSV-tagged MC159 FLIP in the various clones. Black lines indicate where lanes have been removed. Similar data were obtained in two independent experiments

three consensus PxQxS/T TRAF-binding motifs ${ }^{33}$ within the last 28 amino acids (Figure 2a). To test whether these consensus motifs are relevant to TRAF binding, MC159 FLIP was coexpressed with different human TRAF expression constructs in 293T cells, and FLAG-TRAF immunoprecipitates were analyzed for the presence of VSV-tagged MC159 FLIP (Figure $2 b$ ). TRAF1, -2 and -3 specifically bound to MC159 FLIP, while no binding to TRAF4, -5 and -6 could be detected under these conditions. To address the relevance of the TRAF consensus-binding motif for the observed interactions, we generated MC159 expression constructs that were either doubly mutated in the first two identical TRAF-binding motifs (MC159 DM) or C-terminally deleted to remove all three potential binding sites (MC159 $\Delta$ ) (Figure 2a). Co-immunoprecipitation experiments using these MC159 FLIP mutants revealed that the first two TRAF-binding motifs were specifically required for TRAF3 binding, while binding of TRAF1 and TRAF2 did not appear to be affected by the mutation or deletion of these TRAF-binding sites under conditions of overexpression (Figure 2c). Constructs with only a single mutated PVQES motif still bound TRAF3 efficiently (data not shown). These data suggest that MC159 FLIP specifically recruits TRAF3 via the two redundant Cterminal PVQES TRAF-binding sites. In contrast, mutation of the first two or deletion of all three C-terminal TRAF-binding sites did not affect the binding of MC159 FLIP to FADD and caspase-8, interactions that depend on the two DEDs ${ }^{24-26,30}$ (Figure $2 \mathrm{~d}$ and $\mathrm{e}$ ).

\section{MC159 FLIP mediates recruitment of TRAF2 and TRAF3 into the Fas DISC}

To test whether MC159 FLIP bound endogenous TRAFs in the context of Fas signaling, we generated stable Jurkat cell lines expressing the wild-type, the deleted and the doubly mutated form of MC159 FLIP and analyzed the cells for MC159 FLIP-associated proteins upon treatment with FcFasL (Figure 3a). MC159 FLIP associated with FADD in a ligand-dependent manner, consistent with its described recruitment to the Fas cytoplasmic domain via FADD. ${ }^{26}$ 


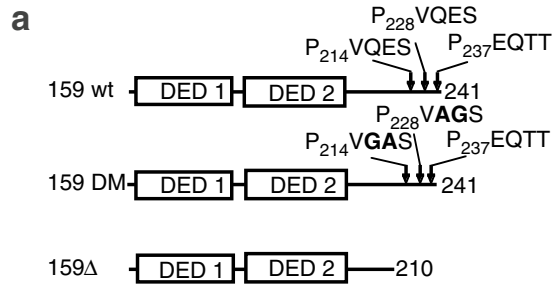

C VSV-FLIP: wt DM $\Delta$ - wt DM $\Delta$ - wt DM $\Delta$ -

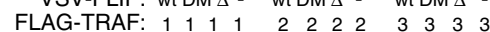
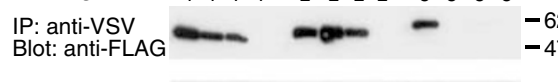

extracts: anti-FLAG

extracts:
anti-VSV

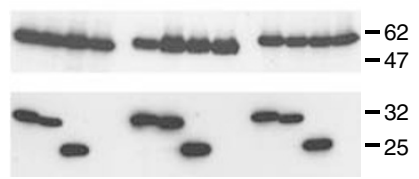

b

FLAG -TRAF: 123456

VSV-FLIP: ++++++

IP: anti-FLAG_- - -32

\begin{tabular}{l|l} 
extracts -32 & Blot: anti-VSV
\end{tabular}

$\mathrm{kDa}$

FLAG -TRAF: $123 \quad 4 \quad 5 \quad 6$

extracts

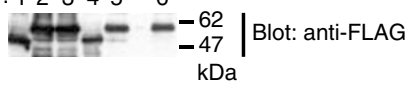

d

\begin{tabular}{|c|c|c|c|c|c|}
\hline $\begin{array}{l}\text { VSV-FLIP: } \\
\text { FADD: }\end{array}$ & $\begin{array}{l}\text { wt DM } \Delta- \\
++++\end{array}$ & & $\begin{array}{l}\text { VSV-FLIP: } \\
\text { aspase-8 (NT): }\end{array}$ & $\begin{array}{l}\text { wt DM } \Delta- \\
++++\end{array}$ & \\
\hline $\begin{array}{l}\text { anti-VSV } \\
\text { :anti-FADD }\end{array}$ & $m=0$ & $\begin{array}{l}-32 \\
-25\end{array}$ & $\begin{array}{l}\text { IP: anti-VSV } \\
\text { Blot: anti-FLAG }\end{array}$ & - & $\begin{array}{l}-32 \\
-25\end{array}$ \\
\hline $\begin{array}{l}\text { cts: } \\
\text { CADD }\end{array}$ & mengen & $\begin{array}{l}-32 \\
-25\end{array}$ & $\begin{array}{l}\text { extracts: } \\
\text { anti-FLAG }\end{array}$ & -- & $\begin{array}{l}-32 \\
-25\end{array}$ \\
\hline $\begin{array}{l}\text { ts: } \\
\text { sv }\end{array}$ & - & -32 & anti & - & 32 \\
\hline & & & & & \\
\hline
\end{tabular}

Figure 2 MC159 FLIP binds TRAF3 via two C-terminal PVQES motifs, (a) Schematic diagram of the MC159 FLIP constructs used in this study: the wild-type form of MC159 FLIP (wt), the double mutant (DM) that is mutated in the two identical PVQES motifs, and the deletion mutant $(\Delta)$ in which the $C$-terminus containing all three potential PXQXS/T consensus sites were removed by Pvull restriction. Numbers indicate amino-acid positions. (b) 293T cells were cotransfected with VSV-tagged MC159 FLIP and the indicated FLAG-tagged TRAF constructs and the binding of FLIP to individual TRAFs was assessed by co-immunoprecipitation. (c) 293T cells were cotransfected with the indicated VSV-tagged MC159 FLIP constructs and FLAG-tagged TRAF1, TRAF2 and TRAF3 constructs and protein-protein interactions were assessed by co-immunoprecipitation. (d, e) 293T cells were cotransfected with the indicated VSV-tagged MC159 FLIP constructs and expression vectors for FADD (d) or for the DED-containing N-terminus of caspase-8 (NT) (e), and the binding of the FLIP mutants to these protein constructs was assessed by co-immunoprecipitation

TRAF2 and TRAF3, on the other hand, bound to wt MC159 FLIP independently of Fas triggering. TRAF1 is very poorly expressed in Jurkat cells, and no binding of endogenous TRAF1 to MC159 FLIP could be detected (data not shown). The double mutant (DM) and the C-terminally deleted form of MC159 FLIP $(\Delta)$ failed to bind to TRAF3, as was previously observed under conditions of overexpression in 293T cells (Figure 2c). TRAF2 still bound to both mutants, although at reduced levels, possibly because TRAF3 contributed to optimal recruitment and/or stabilization of endogenous TRAF2 in the complex. Next, we analyzed whether the different MC159 FLIP constructs allowed the recruitment of TRAF2 and -3 into the Fas DISC in these clones (Figure $3 b$ ). In the presence of the wt form of MC159 FLIP, TRAF2 and TRAF3 were recruited to the Fas DISC in a FasL-dependent manner. TRAF3 recruitment was detectable only in the presence of intact MC159 FLIP. TRAF2 recruitment, on the other hand, was detectable in presence of intact MC159 FLIP or its double mutant, but only weakly in the clone expressing the deletion mutant and very poorly in the mock control. In contrast, the recruitment of FADD was not affected by the presence of the different MC159 FLIP mutants. Caspase-8 recruitment was detectable in the DISC of each cell line $5 \mathrm{~min}$ after FasL stimulation, even though the amount of caspase-8 recruitment was significantly higher in the mock-transfected cells than in the clones expressing the different MC159 FLIP constructs. Moreover, in striking contrast to the mocktransfected clone, no caspase- 8 processing occurred in the DISC of clones expressing different forms of MC159 FLIP during the $45 \mathrm{~min}$ of stimulation. Together, these data suggest that the C-terminal part of MC159 FLIP is required for constitutive binding of TRAF3 and, to a lesser extent, of TRAF2, and for the corresponding inducible recruitment of these TRAF proteins into the Fas DISC.

\section{MC159 FLIP inhibits Fas-induced apoptosis by TRAF-dependent and -independent mechanisms}

Next, we tested whether the different MC159 FLIP mutants were comparable in their ability to inhibit Fas-induced cell death. Expression of MC159 FLIP wt completely protected Jurkat cells against Fas-induced cell death, while the TRAFbinding-deficient deletion and double mutants showed only partial protection against Fas-induced cell death. However, complete protection could be restored by addition of the pancaspase inhibitor z-VAD (Figure 4a). Together with the results described above, these data suggest that the recruitment of TRAF3, and potentially of TRAF2, by MC159 FLIP contributes to the antiapoptotic, but not the antinecrotic properties of MC159 FLIP, most likely by TRAF-dependent inhibition of a z-VAD-sensitive caspase. Similar results were obtained for at least two independent clones of each construct (data not shown), demonstrating that the reduced cell death protection by the mutant constructs was not a clonal artefact, nor due to possible differences in the MC159 FLIP expression levels of the various clones. The specificity of the observation was further underlined by the fact that the different clones were equally susceptible to cell death induction by staurosporine (Figure 4b), a broad range kinase inhibitor that induces apoptosis by mechanisms that are independent of death receptor activation. ${ }^{34}$

FasL-induced activation of caspase- 8 and cleavage of the proapoptotic $\mathrm{Bcl}-2$ family member Bid induces the release of proapoptotic factors from the mitochondrial intermembrane space into the cytosol, which correlates with a loss of the electrochemical potential across the mitochondrial inner membrane $\left(\Delta \Psi_{\mathrm{m}}\right)$. To test the effect of the different forms of MC159 FLIP on FasL- or staurosporine-induced $\Delta \Psi_{\mathrm{m}}$ loss, we analyzed the Jurkat clones for mitochondrial depolarization 
a

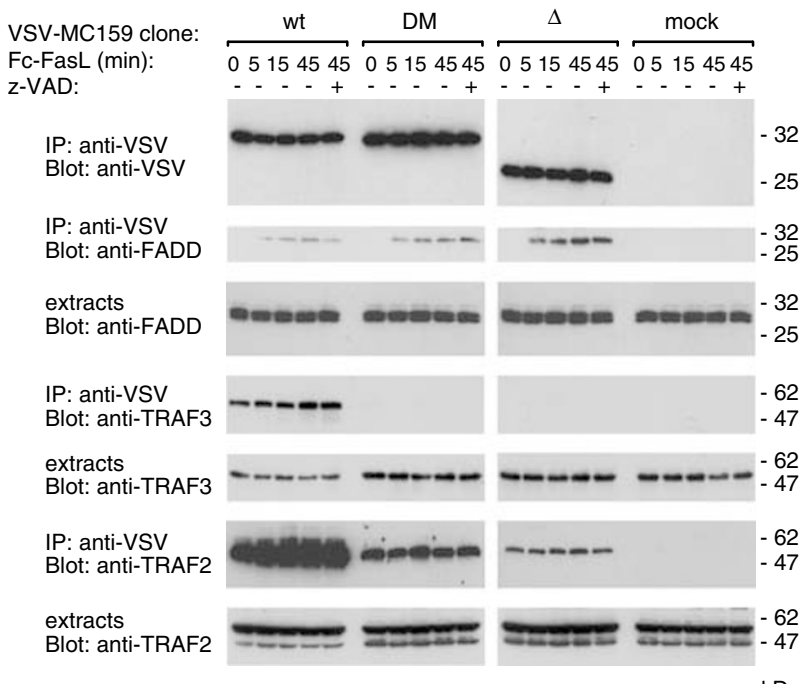

b

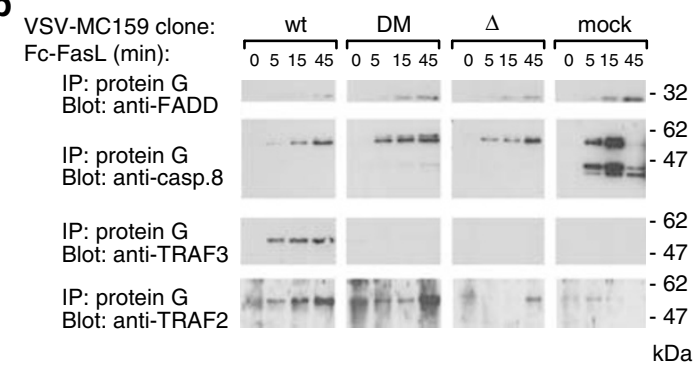

Figure 3 MC159 FLIP mediates recruitment of TRAF2 and -3 into the Fas DISC. (a, b) VSV-tagged MC159 FLIP-expressing Jurkat cell clones were treated with Fc-FasL, as indicated, and (a) anti-VSV immunoprecipitates and cell extracts or (b) protein G-sepharose precipitates containing the Fas-DISC were analyzed for the presence of FADD, caspase-8, TRAF2 and TRAF3 by Western blot. In the control sample, Fc-FasL was added into the lysate of nonstimulated cells at $4^{\circ} \mathrm{C}$. Samples in each horizontal row of panels were electrophoresed, transferred, revealed and exposed simultaneously, but samples of the wt and DM clone were run on one gel, while samples from the $\Delta$ and mock clone were loaded on a second gel. Data shown are representative of three independent experiments, which all yielded comparable results

using TMRM, a potentiometric dye that shows increased mitochondrial uptake and fluorescence intensity in proportion to increased $\Delta \Psi_{\mathrm{m}} \cdot{ }^{35} \mathrm{~A}$ marked difference between the clones was seen when the cells were treated overnight with Fc-FasL, which induced a loss of $\Delta \Psi_{\mathrm{m}}$ in $89 \%$ of the mock-transfected clone, while the MC159 FLIP wt-transfected clone was almost completely protected and lost $\Delta \Psi_{\mathrm{m}}$ in only a very low percentage of the cells (Figure 4c). Interestingly, Jurkat clones expressing the TRAF3-binding-deficient MC159 FLIP double mutant (DM) or the MC159 FLIP deletion mutant $(\Delta)$ showed an average loss of $\Delta \Psi_{\mathrm{m}}$ in 39 and $51 \%$ of cells, respectively, suggesting that the TRAF-binding deficient MC159 FLIP mutants provided less-efficient protection than the wild-type form. The impaired protective effect of the TRAF-binding-mutant forms of FLIP became evident only after prolonged $(16 \mathrm{~h})$ treatment of the cells with Fc-FasL, while at a shorter $(4 \mathrm{~h}$ ) time point the wt and mutant FLIP forms provided similar and almost complete protection (data not
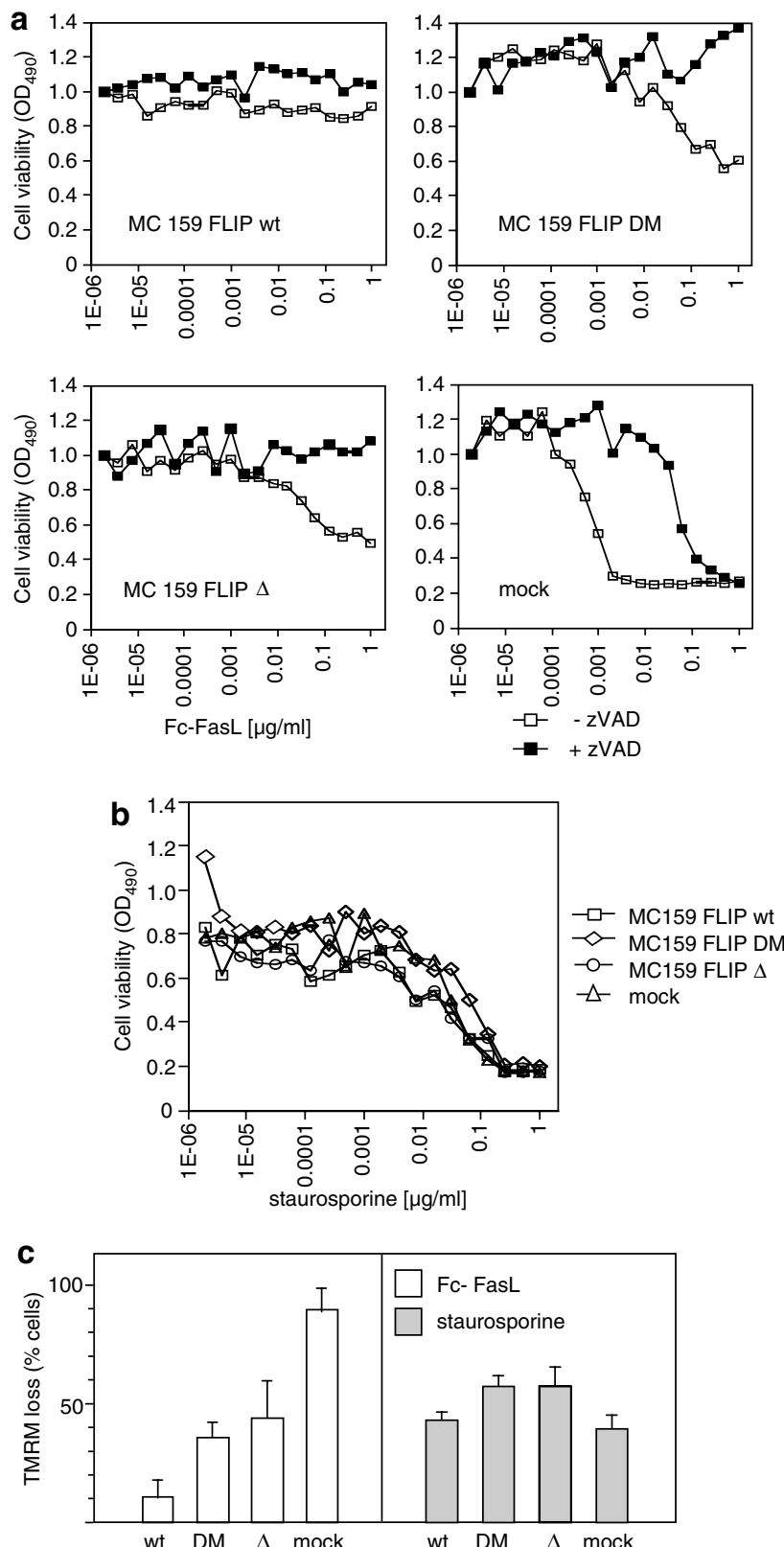

Figure 4 Full inhibition of Fas-induced apoptosis by MC159 FLIP depends on its intact TRAF2/3-binding site. (a) Jurkat clones stably transfected with the indicated MC159 FLIP constructs were treated overnight with Fc-FasL in the presence or absence of Z-VAD and cell viability was assessed using the PMS/ MTS assay. (b) As in (a), but cell death was induced by staurosporine treatment. Comparable results were obtained for at least two independent clones for each MC159 FLIP construct. (c) Jurkat clones stably transfected with the different MC159 FLIP constructs were treated for $4 \mathrm{~h}$ with staurosporine $(2 \mu \mathrm{M})$ or for $16 \mathrm{~h}$ with Fc-FasL $(1 \mu \mathrm{g} / \mathrm{ml})$, and the percentage of cells that lost $\Delta \Psi_{\mathrm{m}}$ was determined by flow cytometry using TMRM. Data shown are mean values \pm standard deviations of two independent experiments carried out in triplicate

shown). In response to a $4 \mathrm{~h}$ staurosporine treatment, on the other hand, a large percentage (40-60\%) of all tested cells showed a loss in the mitochondrial membrane potential (Figure 4c), again indicating that all clones had intact intracellular cell death pathways and that the differences 
observed following Fc-FasL treatment are most likely due to receptor-proximal events. The protective effect of the MC159 FLIP-TRAF interaction appeared to depend on the activity of (a) caspase(s), since addition of the pan-caspase inhibitor z-VAD was able to completely protect the cells from FcFasL-induced loss in $\Delta \Psi_{\mathrm{m}}$ in all clones (data not shown). Collectively, these data suggest that TRAF3- (and possibly TRAF2 co-) recruitment by MC159 FLIP efficiently delays apoptosis by slowing down a critical event of the Fas-signaling pathway at a caspase-dependent step upstream of mitochondria.

\section{The intact TRAF3-binding site of MC159 FLIP is necessary to fully inhibit Fas internalization}

An event that occurs downstream of Fas DISC formation and that depends on caspase activity is the internalization of the engaged receptor. ${ }^{36}$ Therefore, we tested whether the TRAF3-binding site of MC159 FLIP was relevant for Fas receptor internalization. In mock-transfected Jurkat cells, about $25 \%$ of the receptor internalized within 60 min upon stimulation with Fc-FasL, while hardly any receptor internalization could be detected over this time period in cells expressing wt or the TRAF-binding-deficient mutants of MC159 FLIP (Figure 5a). Upon prolonged treatment (up to $16 \mathrm{~h}$ ) with Fc-FasL, on the other hand, a significant percentage of the FLIP double mutant or deletion mutant clones had internalized the receptor ( 30 and $28 \%$, respectively), while minimal Fas internalization (4\%) could be detected in the presence of the wt form of MC159 FLIP (Figure 5b). The percentage of cells that had internalized the receptor correlated well with the percentage of annexin V-positive, apoptotic cells in the same experiments, suggesting that the TRAF3-binding-dependent capacity of MC159 FLIP to inhibit receptor internalization accounted for its antiapoptotic effect (Figure 5c). Together, these data suggest that the intact TRAF3-binding site of MC159 FLIP is required to fully prevent ligand-induced Fas internalization and apoptosis.

\section{TRAF3 binding to MC159 FLIP is necessary to completely block Fas-induced activation of caspases}

Finally, to check whether biochemical differences between the clones could be detected under the conditions of prolonged FasL stimulation used above (Figures 4 and 5), we analyzed cell extracts of Fc-FasL-treated Jurkat clones for the processing of caspase family members in a time course experiment (Figure 6). The wild-type form of MC159 FLIP completely inhibited Fas-induced processing of procaspase-8 and -10 during at least $22 \mathrm{~h}$ of stimulation, while the two TRAF-binding mutants allowed the formation, after $8 \mathrm{~h}$ of stimulation, of previously described caspase- 8 p43/p45 and caspase-10 p43/47 cleavage fragments that result from proteolytic removal of the C-terminal small catalytic subunit. ${ }^{37,38}$ Differences between the inhibitory capacity of the wt and mutant forms of MC159 were even more striking in the case of the executioner caspases, caspase- 3 and -9 , probably because of signal amplification downstream of caspase-8. Upon a $1 \mathrm{~h}$

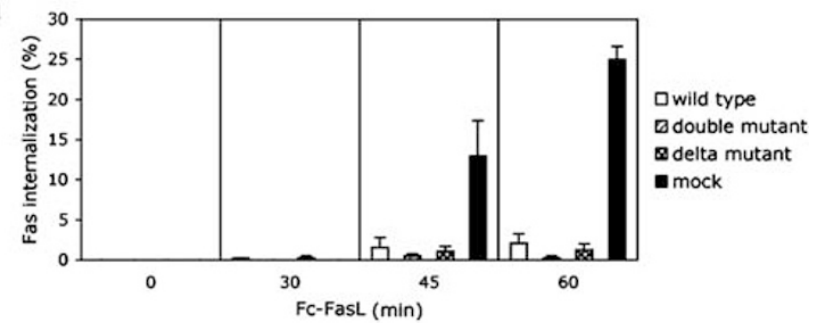

b

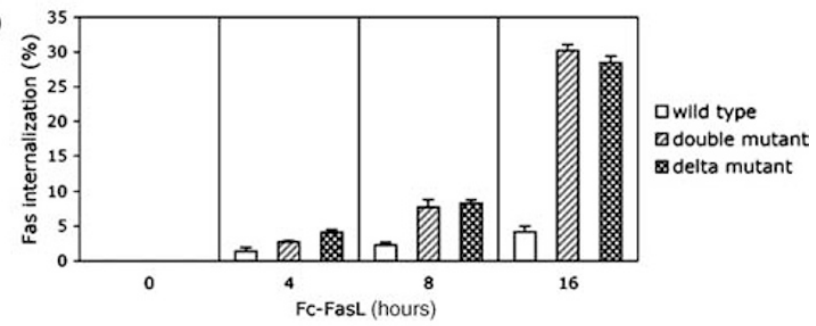

C

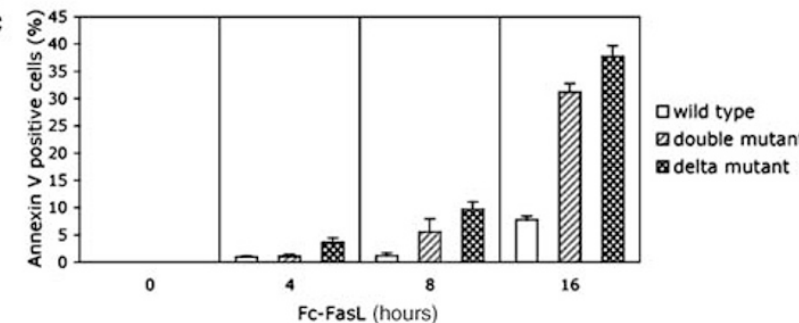

Figure 5 The intact TRAF3-binding site of MC159 FLIP is required for efficient inhibition of receptor internalization. Jurkat clones stably transfected with the different MC159 FLIP constructs or mock vector were treated with Fc-FasL and the percentage of cells that had lost surface Fas over (a) $60 \mathrm{~min}$ or (b) $16 \mathrm{~h}$ or that had become annexin $\mathrm{V}$ positive over $16 \mathrm{~h}$ (c) was assessed by flow cytometry. Data shown are representative of several determinations and values were calculated as mean values \pm standard deviations of three independent experiments

treatment with Fc-FasL, caspase-3 and -9 were completely processed in the mock-transfected Jurkat cells, while Fc-FasL treatment for up to $22 \mathrm{~h}$ did not induce significant caspase-3 and -9 processing in the Jurkat cells expressing wt MC159. In the clones expressing the MC159 mutants, the total amounts of procaspase-3 in the cell extracts progressively diminished. The clones also showed a small reduction in the total amounts of procaspase- 9 that correlated with the generation of a fragment corresponding to active caspase- 9 (p37) that became detectable after $8 \mathrm{~h}$ of stimulation. Together, these data support the idea that the intact TRAF2/3-binding site of MC159 FLIP is crucial to fully prevent FasL-induced activation of initiator and effector caspases during sustained Fas triggering.

\section{Discussion}

MCV is a virus that efficiently evades the immune system of the infected host, because it encodes proteins that either directly increase the resistance of the host cell to apoptosis or target other aspects of the host immune response. ${ }^{18,23}$ This allows the virus to persist for months in the skin of infected 


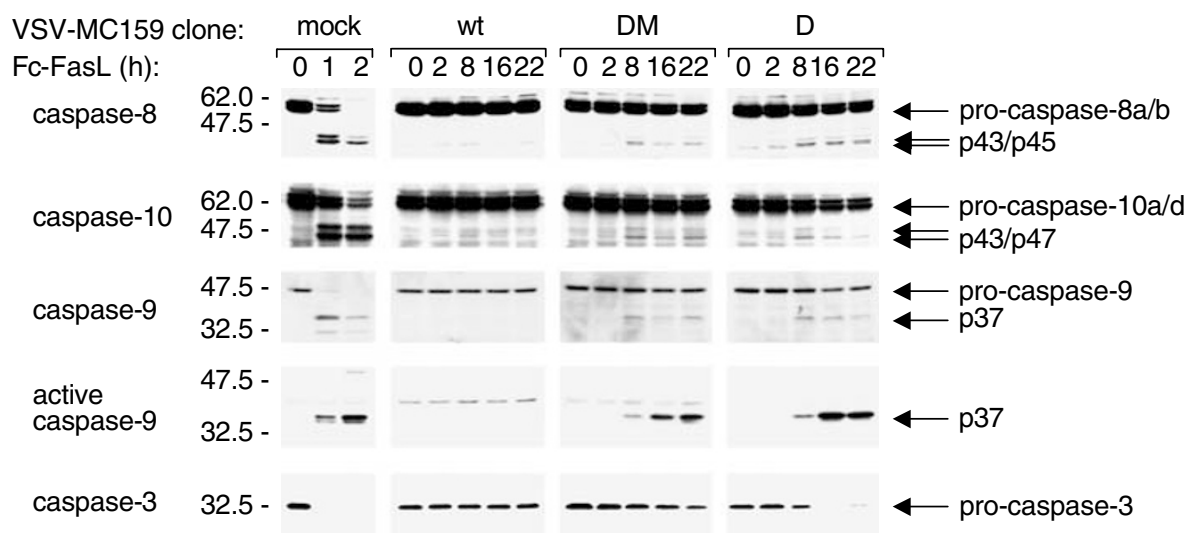

Figure 6 The intact TRAF3-binding site of MC159 FLIP is required for complete and extended inhibition of caspase-8 processing. Jurkat clones stably transfected with the different MC159 FLIP constructs were treated for the indicated times with Fc-FasL and thereafter processing of caspase-8, $-10,-9$ and -3 was assessed by Western blotting of cell extracts using the indicated primary antibodies. Panels represent images that were acquired simultaneously, but samples of the wt and DM clone were run on one gel, while samples from the $\Delta$ and mock clone were loaded on a second gel. All samples were electrophoresed, transferred, revealed and exposed in parallel

individuals without signs of inflammation, and to efficiently escape elimination by CTLs. Our study suggests that MC159 FLIP contributes to the particularly efficient protection of the virus-infected host cell by multiple mechanisms, including the extended inhibition of both caspase-dependent and caspaseindependent Fas-induced cell death. In particular, we show that the MC159 FLIP C-terminal region contains TRAF consensus binding sites that are critical for binding to TRAF3 and contribute to optimal binding of TRAF2. These binding sites are necessary for optimal recruitment of both TRAF family members to the DISC, and for full apoptosis protection under conditions of prolonged FasL stimulation, but do not affect the MC159-mediated protection against Fas-induced necrosis, since addition of z-VAD restored full cell death protection of the TRAF-binding-deficient MC159 FLIP mutants.

Mutation or deletion of the two MC159 TRAF-binding consensus sites fully impaired binding of TRAF3, but had only a partial effect on TRAF2 binding, suggesting that MC159 contains yet another TRAF2-binding motif. Interestingly, a recent study showed that TRAF2 binds to the first DED of MC159 under conditions of overexpression. ${ }^{39}$ It is thus possible that optimal TRAF2 binding requires both its interaction with the first DED of MC159 and an interaction with MC159-bound TRAF3, which has been previously shown to form heterotrimers with TRAF2. ${ }^{40}$

The molecular function of TRAF2 and TRAF3 in the context of MC159-mediated apoptosis protection is presently ill defined. TRAF family members act as adaptor or scaffolding proteins that transmit signals emanating from TNFR family members either by activation of the NF- $\kappa \mathrm{B}$, JNK and p38 transcriptional pathways or by transcription-independent pathways. ${ }^{33,41}$ Activation of $\mathrm{NF}-\kappa \mathrm{B}$ can protect against apoptosis by inducing the expression of various antiapoptotic genes, ${ }^{42}$ and we thus considered the possibility that this contributed to the TRAF-dependent antiapoptotic properties of MC159. However, the antiapoptotic effect of MC159 observed in this study is unlikely to depend on TRAF-induced protective NF- $\kappa \mathrm{B}$ activation for several reasons. First, pretreatment of the cells with a low nontoxic concentration of $\mathrm{CHX}(0.5 \mu \mathrm{g} / \mathrm{ml})$, reported by others to sensitize Hela and EL4 cells, ${ }^{43}$ did not sensitize the MC159-expressing cells to Fas-induced apoptosis, while at the same concentration it sensitized the mock-transfected Jurkat cells (data not shown). Moreover, no significant NF- $\kappa$ B induction was observed upon transient transfection of MC159 FLIP constructs into Jurkat cells, nor upon FasL treatment of Jurkat clones stably expressing the wt or mutated MC159 constructs used in this study (data not shown). Finally, transient transfection of MC159 FLIP constructs into 293T cells induced only minimal $\mathrm{NF}-\kappa \mathrm{B}$ activation in reporter assays, in contrast to expression of the short and long isoforms of cellular FLIP or of the RIPfamily kinase RIP4, which induced significant NF- $k$ B activation (Supplementary Figure 1).

These findings are consistent with previous reports showing that MC159 acts rather as an inhibitor of NF- $\kappa$ B activation induced by $\mathrm{TNF} \alpha$ treatment ${ }^{39}$ or mediated by transient expression of PKR and caspase-8 and its homologues. ${ }^{44,45}$ By potently inhibiting the death of the infected cell while preventing NF- $\kappa \mathrm{B}$-dependent proinflammatory responses, MC159 may thus assure the prolonged virus survival in the host cell that is characteristic of MCV.

We also considered the possibility that the TRAFs have a proapoptotic role themselves, in the context of Fas signaling, which is inhibited by MC159 FLIP through their sequestration. TRAFs are characterized by the presence of an N-terminal RING finger, a domain that is typically found in proteins with ubiquitin ligase activity. ${ }^{46}$ For TRAF3, a direct proapoptotic role downstream of the $\mathrm{LT} \beta$ receptor has been proposed based on cellular studies using a DN TRAF3 construct lacking the RING finger, ${ }^{47-49}$ but the molecular mechanism underlying this proapoptotic function of TRAF3 remains unknown. We have attempted to interfere with the TRAF3-dependent antiapoptotic function of MC159 FLIP through the use of DN-TRAF3 constructs affecting the ubiquitin ligase activity of the RING finger, ${ }^{50}$ but did not observe any significant changes in Fasinduced apoptosis in the different clones (data not shown), 
suggesting that the antiapoptotic capacity of the TRAF3binding MC159 FLIP does not depend on TRAF3 ubiquitin ligase activity.

Receptor activation by agonistic antibody or recombinant oligomeric FasL has been shown to trigger Fas internalization by mechanisms that depend on caspase- 8 activity. ${ }^{36}$ The wild-type form of MC159, but not its TRAF3-binding-deficient mutants, efficiently inhibited both receptor endocytosis and caspase-8 processing upon elongated Fas stimulation. It remains, however, to be determined whether the MC159 FLIP/TRAF2/3 complex inhibits Fas endocytosis as a consequence of inhibiting caspase-8 activation, or whether the receptor-bound MC159 FLIP/TRAF2/3 complex interferes with caspase-8 processing by directly inhibiting receptor endocytosis, for example, by interaction with DISC-associated proteins that may target the receptor for endocytosis. ${ }^{51}$ Binding of TRAF2 and -3 to CD40 has indeed been shown to be required for ligand-induced downmodulation of this receptor, possibly by TRAF2-dependent recruitment to lipid rafts and subsequent TRAF3-dependent receptor internalization. $^{52}$

Binding to TRAF2 and TRAF3 has been reported not only for MC159 but also for the long form of cellular FLIP, C-FLIP , under conditions of overexpression, ${ }^{53,54}$ and increased recruitment of TRAF2 to the Fas DISC was observed in Jurkat and Raji cells upon stable transfection with CFLIPL. $^{53}$ While this suggests that both $\mathrm{MC} 159$ and CFLIP $\mathrm{P}_{\mathrm{L}}$ interfere with the function of TRAF2, it is unclear whether C-FLIPL also recruits endogenous TRAF3 to the DISC and if so, whether this has a similar or rather an opposing impact on TRAF2 and TRAF3 function. In our hands, MC159 FLIP inhibits Fasinduced cell death in Jurkat cells much more powerfully than CFLIP ${ }_{L}{ }^{26}$ This may be related to TRAF-independent mechanisms, such as the fact that $C F L I P_{L}$ does not inhibit the processing of caspase- 8 at the DISC ${ }^{55-57}$ but rather seems to favor the partial cleavage and activation of caspase- 8 and subsequent cleavage of local substrates in the proximity of the DISC $^{56}$ and/or induction of apoptosis, ${ }^{55}$ depending on the concentration of $\mathrm{C}-\mathrm{FLIP}_{\mathrm{L}}$ and on the experimental system.

Future studies should provide a better understanding of the specific roles of the different TRAF family members and yield valuable insight into the particular function of TRAF2 and TRAF3 in the context of cell survival mediated by MC159.

\section{Materials and Methods}

\section{Expression vectors}

The expression vector for wt MC159 FLIP with an N-terminal vesicular stomatitis virus (VSV)-tag has been described previously. ${ }^{26} \mathrm{~A}$ deletion construct of MC159 FLIP (MC159 FLIP $\Delta$ ) lacking the 31 C-terminal amino acids and the double mutant (MC159 FLIP DM) with alterations in two consensus TRAF-binding sites ( $P V Q_{216} E S \geqslant P V G A S$ and $P V Q_{230} E S \geqslant P$ VAGS) were obtained by PCR-based mutagenesis using cloned fulllength, wild-type MC159 FLIP CDNA together with standard PCR conditions and Pwo polymerase (Roche). Amplified products were verified by sequencing in both directions and subcloned into expression vectors derived from pCR-3 (Invitrogen) to yield expression constructs with an $\mathrm{N}$-terminal VSV-tag. The expression vectors for FLAG-tagged TRAFs have been described. ${ }^{58}$ The cloning vector $S R \alpha$-puro was a gift of $R$ Sékaly (Montreal, Canada).

\section{Cell lines and culture conditions}

293T cells were grown in Dulbecco's modified Eagle's medium and Jurkat cells were grown in RPMI 1640 medium, both supplemented with $10 \%$ heat-inactivated FCS and penicillin/streptomycin $(100 \mu \mathrm{g} / \mathrm{ml}$ of each).

\section{Transient 293T cell transfection, immunoprecipitation and Western blot analysis}

Transient transfection of 293T cells, cell lysis and co-immunoprecipitation of tagged proteins were performed essentially as described before. ${ }^{26,58}$ Antibodies used for Western blotting include monoclonal anti-FLAG M2 and anti-VSV P5D4, rabbit anti-FLAG and anti-VSV antibodies (Sigma), monoclonal anti-FADD (BD Biosciences), rabbit anti-TRAF2 and antiTRAF3 (C-20 and H-122, Santa Cruz), monoclonal anti-caspase-3 (clone 19, Transduction Labs), rabbit anti-caspase-9 (ProSci), rabbit anti-active caspase-9 (Cell Signaling), monoclonal anti-caspase-10 (4C1, MBL) and monoclonal anti-caspase-8 (5F7, MBL). Primary antibodies were revealed using horseradish peroxidase-coupled goat anti-mouse or goat anti-rabbit secondary antibodies (Jackson Immunoresearch) or isotype-specific goat anti-mouse antibodies (Southern Biotechnology).

\section{Stable transfection of Jurkat cells}

For generation of stable Jurkat clones expressing wt or mutant forms of MC159 FLIP, N-terminally VSV-tagged MC159 FLIP constructs were subloned into $\mathrm{SR} \alpha$-puro, an expression vector with $\mathrm{SR} \alpha$ promoter and puromycin selection. 3-5 $\times 10^{6}$ exponentially grown Jurkat cells were transfected with $4 \mu \mathrm{g}$ of SR $\alpha$-puro MC159 FLIP vector or empty vector by electroporation using the Amaxa Nucleofector system (solution V, program 0-17) and stable clones were selected in RPMI complete medium with $5 \mu \mathrm{g} / \mathrm{ml}$ of puromycin (Sigma).

\section{Cell viability assay}

Jurkat cells (seeded at 50000 cells $/ 100 \mu$ in 96 -well plates) were treated with the indicated concentrations of staurosporine or Fc-FasL ${ }^{32}$ (a kind gift of Pascal Schneider) overnight, and cell viability was subsequently assessed using the Celltiter $96 \mathrm{AQ}$ (PMS/MTS) proliferation assay (Promega).

\section{Fas DISC analysis and MC159 FLIP co-immunoprecipitation assays}

Jurkat clones $\left(5 \times 10^{7} / \mathrm{ml}\right)$ were stimulated with or without $1 \mu \mathrm{g} / \mathrm{ml}$ of FcFasL for the indicated times, washed once with ice-cold PBS and lysed in $1 \mathrm{ml}$ of lysis buffer containing $20 \mathrm{mM}$ Tris $\mathrm{pH} 7.4,150 \mathrm{mM} \mathrm{NaCl}, 10 \%$ glycerol, $0.2 \%$ NP40 and protease inhibitors (complete, Roche) on ice for $15 \mathrm{~min}$. Subsequently, samples were subjected to centrifugation at $15000 \times g$ at $4^{\circ} \mathrm{C}$ for $10 \mathrm{~min}$, and $0.25 \mu \mathrm{g} \mathrm{Fc-FasL}$ was added into the lysate of nontreated samples. A small aliquot of postnuclear lysates was used directly for protein analysis by Western blot, while the remaining lysate was precleared with Sepharose $6 \mathrm{~B}$ beads for $1 \mathrm{~h}$ at $4^{\circ} \mathrm{C}$ with constant agitation. Subsequently, Fc-FasL and associated proteins were recovered by addition of protein $G$ Sepharose (Amersham Biosciences) and incubation for at least $4 \mathrm{~h}$ at $4^{\circ} \mathrm{C}$ with constant agitation. Finally, beads 
were washed three times with lysis buffer before boiling in reducing DTT-containing sample buffer and analysis by SDS-PAGE and Western blot.

\section{Measurement of $\Delta \Psi_{\mathrm{m}}$ in Jurkat cells}

Jurkat clones (approximately $5 \times 10^{5}$ cells) were treated with $2 \mu \mathrm{M}$ staurosporine for $4 \mathrm{~h}$ or $1 \mu \mathrm{g} / \mathrm{ml}$ of Fc-FasL for $16 \mathrm{~h}$, and then tetramethyl rhodamine methyl ester (TMRM, Molecular Probes) was added to the cells at a concentration of $0.1 \mu \mathrm{M}$ during the final $15 \mathrm{~min}$ of incubation. TMRM is a mitochondrion-specific potentiometric dye that shows increased mitochondrial uptake and fluorescence intensity in proportion to increased $\Delta \Psi_{\mathrm{m} .}{ }^{35}$ Fluorescence was analyzed by flow cytometry. For each sample, a total of 25000 cells were analyzed with fluorescence signals at logarithmic scale. The reduction in the percentage of TMRM-positive cells in treated versus control cells was calculated for the ungated cell population. The results of two independent experiments performed in triplicate $( \pm$ S.D.) are shown.

\section{Measurement of Fas endocytosis and annexin V staining}

Jurkat cells (approximately $5 \times 10^{5}$ cells) were incubated with $1 \mu \mathrm{g} / \mathrm{ml}$ of Fc-FasL for $15 \mathrm{~min}$ at $4^{\circ} \mathrm{C}$. Fas endocytosis was initiated by shifting the cells to $37^{\circ} \mathrm{C}$ and aliquots were removed, placed on ice at the indicated times and treated with PBS containing $0.1 \%$ azide. Subsequently, cell surface Fas was quantified by staining with FITC-labeled anti-Fas (APO-11, Apotech Corp.) and flow cytometry. The percentage of cells with internalized Fas was calculated by subtracting the percentage of cells that were positive for cell surface Fas at various times post treatment from the percentage of positive cells in samples harvested at time zero.

In order to monitor apoptosis, cells $\left(5 \times 10^{5}\right.$ per sample) were washed twice in Annexin V-binding buffer (10 mM HEPES/NaOH pH 7.4, $140 \mathrm{mM}$ $\mathrm{NaCl}, 2.5 \mathrm{mM} \mathrm{CaCl}_{2}$ ) at $4^{\circ} \mathrm{C}$. They were then resuspended in $100 \mu \mathrm{l}$ binding buffer containing Annexin V-FITC (Alexis) diluted $1: 20$. Samples were incubated at room temperature for $15 \mathrm{~min}$, washed twice in binding buffer and fluorescent staining was determined by flow cytometry.

\section{Acknowledgements}

We thank Daniel Legler, Fabio Martinon, Olivier Micheau and Pascal Schneider for discussions and comments on the manuscript. This work was supported by grants of the Swiss Cancer League and the Swiss National Science Foundation to M Thome and J Tschopp, by a research fellowship of the Deutsche Forschungsgesellschaft to $M$ Thurau, and by an EMBO long-term fellowship to $\mathrm{H}$ Everett.

\section{References}

1. Chipuk JE and Green DR (2005) Opinion: do inducers of apoptosis trigger caspase-independent cell death? Nat. Rev. Mol. Cell. Biol. 6: 268-275

2. Jaattela $M$ and Tschopp $J$ (2003) Caspase-independent cell death in $T$ lymphocytes. Nat. Immunol. 4: 416-423

3. Krammer PH (2000) CD95's deadly mission in the immune system. Nature 407: 789-795

4. Peter ME and Krammer PH (2003) The CD95(APO-1/Fas) DISC and beyond. Cell. Death Differ. 10: 26-35

5. Boatright KM, Renatus M, Scott FL, Sperandio S, Shin H, Pedersen IM, Ricci JE, Edris WA, Sutherlin DP, Green DR and Salvesen GS (2003) A unified model for apical caspase activation. Mol. Cell 11: 529-541
6. Donepudi M, Mac Sweeney A, Briand C and Grutter MG (2003) Insights into the regulatory mechanism for caspase-8 activation. Mol. Cell 11: 543-549

7. Chang DW, Xing Z, Capacio VL, Peter ME and Yang X (2003) Interdimer processing mechanism of procaspase-8 activation. EMBO J. 22: 4132-4142

8. Stennicke HR, Jurgensmeier JM, Shin H, Deveraux Q, Wolf BB, Yang X, Zhou $Q$, Ellerby HM, Ellerby LM, Bredesen D, Green DR, Reed JC, Froelich CJ and Salvesen GS (1998) Pro-caspase-3 is a major physiologic target of caspase-8. J. Biol. Chem. 273: 27084-27090

9. Scaffidi C, Fulda S, Srinivasan A, Friesen C, Li F, Tomaselli KJ, Debatin KM, Krammer PH and BPeter ME (1998) Two CD95 (APO-1/Fas) signaling pathways. EMBO J. 17: 1675-1687

10. Li H, Zhu H, Xu CJ and Yuan J (1998) Cleavage of BID by caspase 8 mediates the mitochondrial damage in the Fas pathway of apoptosis. Cell 94: 491-501

11. Luo X, Budihardjo I, Zou H, Slaughter C and Wang X (1998) Bid, a Bcl2 interacting protein, mediates cytochrome $c$ release from mitochondria in response to activation of cell surface death receptors. Cell 94: 481-490

12. Korsmeyer SJ, Wei MC, Saito M, Weiler S, On KJ and Schlesinger PH (2000) Pro-apoptotic cascade activates BID, which oligomerizes BAK or BAX into pores that result in the release of cytochrome $c$. Cell Death Differ. 7: 1166-1173

13. Li P, Nijhawan D, Budihardjo I, Srinivasula SM, Ahmad M, Alnemri ES and Wang X (1997) Cytochrome $c$ and dATP-dependent formation of Apaf-1/ caspase-9 complex initiates an apoptotic protease cascade. Cell 91: 479-489

14. Zou H, Li Y, Liu X and Wang B (1999) An APAF-1. cytochrome $c$ multimeric complex is a functional apoptosome that activates procaspase-9. J. Biol. Chem. 274: 11549-11556

15. Acehan D, Jiang X, Morgan DG, Heuser JE, Wang X and Akey CW (2002) Three-dimensional structure of the apoptosome: implications for assembly, procaspase-9 binding, and activation. Mol. Cell 9: 423-432

16. Holler N, Zaru R, Micheau O, Thome M, Attinger A, Valitutti S, Bodmer J-L, Schneider P, Seed B and Tschopp J (2000) Fas triggers an alternative, caspase-8-independent cell death pathway using the kinase RIP as effector molecule. Nat. Immunol. 1: 489-495

17. Stanger BZ, Leder P, Lee TH, Kim E and Seed B (1995) RIP: a novel protein containing a death domain that interacts with Fas/APO-1 (CD95) in yeast and causes cell death. Cell. 81: 513-523

18. Moss B, Shisler J, Xiang $Y$ and Senkevich TG (2000) Immune-defense molecules of Molluscum contagiosum virus, a human poxvirus. Trends Microbiol. 8: 473-477

19. Siegel RM, Chan FK, Chun HJ and Lenardo MJ (2000) The multifaceted role of Fas signaling in immune cell homeostasis and autoimmunity. Nat. Immunol. 1: 469-474

20. Tschopp J, Thome M, Hofmann K and Meinl E (1998) The fight of viruses against apoptosis. Curr. Opin. Genet. Dev. 8: 82-87

21. Everett $H$ and McFadden $G$ (2002) Poxviruses and apoptosis: a time to die. Curr. Opin. Microbiol. 5: 395-402

22. Thome M and Tschopp J (2001) Regulation of lymphocyte proliferation and death by FLIP. Nat. Rev. Immunol. 1: 50-58

23. Smith KJ, Yeager $\mathrm{J}$ and Skelton $\mathrm{H}$ (1999) Molluscum contagiosum: its clinical, histopathologic, and immunohistochemical spectrum. Int. J. Dermatol. 38: 664-672

24. Hu S, Vincenz C, Buller M and Dixit VM (1997) A novel family of viral death effector domain-containing molecules that inhibit both CD-95- and tumor necrosis factor receptor-1-induced apoptosis. J. Biol. Chem. 272: 9621-9624

25. Bertin J, Armstrong RC, Ottilie S, Martin DA, Wang Y, Banks S, Wang GH, Senkevich TG, Alnemri ES, Moss B, Lenardo MJ, Tomaselli KJ and Cohen JI (1997) Death effector domain-containing herpesvirus and poxvirus proteins inhibit both Fas-and TNFR1-induced apoptosis. Proc. Natl. Acad. Sci. U.S.A. 94: $1172-1176$

26. Thome M, Schneider $P$, Hofmann K, Fickenscher H, Meinl E, Neipel F, Mattmann C, Burns K, Bodmer JL, Schroter M, Scaffidi C, Krammer PH, Peter ME and Tschopp J (1997) Viral FLICE-inhibitory proteins (FLIPs) prevent apoptosis induced by death receptors. Nature 386: $517-521$

27. Shisler JL and Moss B (2001) Molluscum contagiosum virus inhibitors of apoptosis: the MC159 V-FLIP protein blocks Fas-induced activation of procaspases and degradation of the related MC160 protein. Virology 282 : 14-25

28. Garvey TL, Bertin J, Siegel RM, Wang GH, Lenardo MJ and Cohen JI (2002) Binding of FADD and caspase-8 to molluscum contagiosum virus MC159 v-FLIP is not sufficient for its antiapoptotic function. J. Virol. 76: 697-706 
29. Bugert JJ, Melquiot N and Kehm R (2001) Molluscum contagiosum virus expresses late genes in primary human fibroblasts but does not produce infectious progeny. Virus Genes 22: 27-33

30. Garvey T, Bertin J, Siegel R, Lenardo M and Cohen J (2002) The death effector domains (DEDs) of the molluscum contagiosum virus MC159 v-FLIP protein are not functionally interchangeable with each other or with the DEDs of caspase-8. Virology 300: 217-225

31. Chan FK, Shisler J, Bixby JG, Felices M, Zheng L, Appel M, Orenstein J, Moss $B$ and Lenardo MJ (2003) A role for tumor necrosis factor receptor 2 (TNFR-2) and receptor-interacting protein (RIP) in programmed necrosis and anti-viral responses. J. Biol. Chem. 278: 51613-51621

32. Holler N, Tardivel A, Kovacsovics-Bankowski M, Hertig S, Gaide O, Martinon F, Tinel A, Deperthes D, Calderara S, Schulthess T, Engel J, Schneider P and Tschopp J (2003) Two adjacent trimeric Fas ligands are required for Fas signaling and formation of a death-inducing signaling complex. Mol. Cell. Biol. 23: $1428-1440$

33. Arch RH, Gedrich RW and Thompson CB (1998) Tumor necrosis factor receptor-associated factors (TRAFs) - a family of adapter proteins that regulates life and death. Genes Dev. 12: 2821-2830

34. Liu X, Kim CN, Yang J, Jemmerson R and Wang X (1996) Induction of apoptotic program in cell-free extracts: requirement for dATP and cytochrome c. Cell 86: 147-157

35. Scaduto RC and Grotyohann LW (1999) Measurement of mitochondrial membrane potential using fluorescent rhodamine derivatives. Biophys. J. 76: $469-477$

36. Algeciras-Schimnich A, Shen L, Barnhart BC, Murmann AE, Burkhardt JK and Peter ME (2002) Molecular ordering of the initial signaling events of CD95. Mol. Cell. Biol. 22: 207-220

37. Medema JP, Scaffidi C, Kischkel FC, Shevchenko A, Mann M, Krammer PH and Peter ME (1997) FLICE is activated by association with the CD95 deathinducing signaling complex (DISC). EMBO J. 16: 2794-2804

38. Kischkel FC, Lawrence DA, Tinel A, LeBlanc H, Virmani A, Schow P, Gazdar A, Blenis J, Arnott D and Ashkenazi A (2001) Death receptor recruitment of endogenous caspase-10 and apoptosis initiation in the absence of caspase-8. J. Biol. Chem. 276: 46639-46646

39. Murao LE and Shisler JL (2005) The MCV MC159 protein inhibits late, but not early, events of TNF-alpha-induced NF-kappaB activation. Virology 340: $255-264$

40. He L, Grammer AC, Wu X and Lipsky PE (2004) TRAF3 forms heterotrimers with TRAF2 and modulates its ability to mediate NF-kB activation. J. Biol. Chem. 279: 55855-55865

41. Chung JY, Park YC, Ye H and Wu $\mathrm{H}$ (2002) All TRAFs are not created equal: common and distinct molecular mechanisms of TRAF-mediated signal transduction. J. Cell Sci. 115: 679-688

42. Wang CY, Mayo MW, Korneluk RG, Goeddel DV and Baldwin Jr AS (1998) NF-kappaB antiapoptosis: induction of TRAF1 and TRAF2 and C-IAP1 and C-IAP2 to suppress caspase-8 activation. Science 281: 1680-1683

43. Micheau O, Lens S, Gaide O, Alevizopoulos K and Tschopp J (2001) NF-kB signals induce the expression of c-FLIP. Mol. Cell. Biol. 21: 5299-5305
44. Chaudhary PM, Jasmin A, Eby MT and Hood L (1999) Modulation of the NF-kappa B pathway by virally encoded death effector domains-containing proteins. Oncogene 18: $5738-5746$

45. Gil J, Rullas J, Alcami $\mathrm{J}$ and Esteban M (2001) MC159L protein from the poxvirus molluscum contagiosum virus inhibits NF-kappaB activation and apoptosis induced by PKR. J. Gen. Virol. 82: 3027-3034

46. Joazeiro CA and Weissman AM (2000) RING finger proteins: mediators of ubiquitin ligase activity. Cell 102: 549-552

47. VanArsdale TL, VanArsdale SL, Force WR, Walter BN, Mosialos G, Kieff E, Reed JC and Ware CF (1997) Lymphotoxin-beta receptor signaling complex: role of tumor necrosis factor receptor-associated factor 3 recruitment in cell death and activation of nuclear factor kappaB. Proc. Natl. Acad. Sci. U.S.A. 94: 2460-2465

48. Force WR, Cheung TC and Ware CF (1997) Dominant negative mutants of TRAF3 reveal an important role for the coiled coil domains in cell death signaling by the lymphotoxin-beta receptor. J. Biol. Chem. 272: 30835-30840

49. Wu MY, Wang PY, Han SH and Hsieh SL (1999) The cytoplasmic domain of the lymphotoxin-beta receptor mediates cell death in HeLa cells. J. Biol. Chem. 274: 11868-11873

50. Rothenberger S, Burns K, Rousseaux M, Tschopp J and Bron C (2003) Ubiquitination of the Epstein-Barr virus-encoded latent membrane protein 1 depends on the integrity of the TRAF binding site. Oncogene 22: $5614-5618$

51. Bonifacino JS and Traub LM (2003) Signals for sorting of transmembrane proteins to endosomes and lysosomes. Annu. Rev. Biochem. 72: 395-447

52. Manning E, Pullen SS, Souza DJ, Kehry M and Noelle RJ (2002) Cellular responses to murine $C D 40$ in a mouse $B$ cell line may be TRAF dependent or independent. Eur. J. Immunol. 32: 39-49

53. Kataoka T, Budd RC, Holler N, Thome M, Martinon F, Irmler M, Burns K, Hahne M, Kennedy N, Kovacsovics M and Tschopp J (2000) The caspase-8 inhibitor FLIP promotes activation of NF-kappaB and Erk signaling pathways. Curr. Biol. 10: $640-648$

54. Shu HB, Halpin DR and Goeddel DV (1997) Casper is a FADD- and caspaserelated inducer of apoptosis. Immunity 6: 751-763

55. Chang DW, Xing Z, Pan Y, Algeciras-Schimnich A, Barnhart BC, Yaish-Ohad $S$, Peter ME and Yang $X(2002) c-F L I P(L)$ is a dual function regulator for caspase-8 activation and CD95-mediated apoptosis. EMBO J. 21: 3704-3714

56. Micheau O, Thome M, Schneider P, Holler N, Tschopp J, Nicholson DW, Briand C and Grutter MG (2002) The long form of FLIP is an activator of caspase-8 at the Fas death-inducing signaling complex. J. Biol. Chem. 277: $45162-45171$

57. Boatright KM, Deis C, Denault JB, Sutherlin DP and Salvesen GS (2004) Activation of caspases-8 and -10 by FLIP(L). Biochem. J. 382: 651-657

58. Thome M, Martinon F, Hofmann K, Rubio V, Steiner V, Schneider P, Mattmann $C$ and Tschopp J (1999) Equine herpesvirus-2 E10 gene product, but not its cellular homologue, activates NF-kappaB transcription factor and C-Jun N-terminal kinase. J. Biol. Chem. 274: 9962-9968

Supplementary Information accompanies the paper on Cell Death and Differentiation website (http://www.nature.com/cdd) 